\title{
Diagnóstico socio-ambiental de la producción agrícola en el páramo de Rabanal (Colombia) como base para su reconversión agroecológica
}

\author{
Socio-environmental diagnostic of the agricultural \\ production on the Rabanal paramo (Colombia) as a \\ basisforits agroecological reset
}

Fecha de recepción: 18 de septiembre de 2014

Fecha de aceptación: 2 de diciembre de 2014

Dalia Soraya Useche de Vega ${ }^{1}$,

Sara María Márquez-Girón²

\section{Resumen}

En las partes más altas de las montañas andinas, entre los límites superior de bosques e inferior de zonas nivales, se encuentran los páramos; en Colombia se localizan en las cordilleras Occidental, Central y Oriental, y en la Sierra Nevada de Santa Marta; abarcan gran extensión del país y tienen máxima representatividad en el departamento de Boyacá, en donde abastecen acueductos municipales y veredales.

Dadas las características geológicas, climáticas y edáficas de los páramos, se han desarrollado en ellos la extracción minera (principalmente carbón) y actividades agropecuarias (principalmente papa y pastoreo de vacunos y ovinos) sobre $3.000 \mathrm{msnm}$; pero las ventajas ofrecidas por este medio natural para desarroIlar estas actividades contrastan con su susceptibilidad al deterioro y la vulnerabilidad edáfica.

La aplicación de agroquímicos y la ampliación de la frontera agropecuaria son actividades que alteran negativamente el ecosistema de páramo, impactan los recursos naturales, la biodiversidad y las fuentes hídricas; pero el enfoque agrícola actual del país no permite analizar las relaciones socio-económicas existentes entre la población, ni las necesidades, ni, mucho menos, las potencialidades de los campesinos asentados allí.

Ante esta problemática se pretende, bajo un enfoque de sistemas ya partir del diagnóstico socioeconómico y ambiental y de la comprensión de la realidad campesina, vincular, mediante estrategias de sensibili-

1 Ph.D (c) en Agroecología de la Universidad de Antioquia. Universidad Pedagógica y Tecnológica de Colombia (Tunja - Boyacá, Colombia).dalia.useche@uptc.edu.co.

2 Ph.D. Universidad de Antioquia (Medellín - Antioquia, Colombia). 
zación, a la comunidad para que participe activamente en la evaluación de la salud del suelo y del cultivo y en la identificación y reconocimiento de la biodiversidad existente, así como de la capacidad de resiliencia del agroecosistema. Todo esto teniendo como principio la agroecología, en la búsqueda de una alternativa socialmente viable y ambientalmente sostenible en este ecosistema de especial protección.

Palabras clave: Agroecosistema, biodiversidad, frontera agropecuaria, páramos, resiliencia del agrosistema.

\begin{abstract}
The paramos are situated in the highest parts of the Andean mountains, between the woods superior limits, and the snow lower layer. In Colombia the paramos are located in the Western, Central and Eastern mountain ranges, as well as in theSanta Marta Sierra Nevada; they cover a large extension of the country, reaching their maximum representativeness in the Boyacá department, where they supply water for the cities and country side aquaducts. The paramo geological, climatic and edaphic characteristics, has attracted the mining development, mainly coal, plus the growing vegetables specially potatos and the establishment of cattle and sheep grazing, over the 3000 masl. The relative advantages offered by the natural environment for the development of these activities, are in contrast with the soil deterioration susceptibility and with the great vulnerability of the altoandinos ecosystems, subject to extreme weather conditions, high humidity and steep slopes.

The application of agrochemicals and the agricultural frontier expansion are activities that affect negatively the paramo ecosystem, impact the natural resources, the biodiversity, and the water sources. But theCountry's current agricultural approach that does not allow to analyze the socio-economic relationships between the population, neither their needs, nor much less the potential of the farmers settled there. Given this problematic, is intended, under a systems approach, and from the socio-economic and environmental assessment, to link the community in a participatory manner, and through sensitization strategies, to become masters of their rural reality, by looking for the soil and crop health valuation, and to identify and recognize the existing biodiversity, also the agroecosystem resilience capacity. All this taking as a principle the agroecology, in search for a socially viable alternative and environmentally sustainable in this today unprotected ecosystem.
\end{abstract}

Key Words: Agroecosystem, Biodiversity, Agricultural Frontier, Paramos, Agrosystem Resilience. 


\section{Introducción}

Históricamente, Colombia ha sufrido grandes impactos sobre sus ecosistemas, lo cual ha generado la transformación de hábitats, más aún con la ampliación de los asentamientos humanos (1), que Ilevan a procesos muy fuertes de pérdida y fragmentación de los ecosistemas nativos. Se estima que más del $66 \%$ de la cobertura vegetal original ha desaparecido, dando lugar a pastizales (2).

La cobertura vegetal que se registra hoy, y que se diezma progresivamente, es menos del 34\%, dominada por los ecosistemas nativos, como bosques, humedales y páramos, los cuales son refugio de alta riqueza y endemismo, sin olvidar altos valores de recambio de especies entre las zonas de vida que componen el territorio nacional (3).

Los páramos juegan un papel importante en la conservación de la biodiversidad, en la regulación del agua y en el almacenamiento de carbono (4); sin embargo, existe una creciente demanda de los centros urbanos de papa y producción de leche, teniendo en cuenta que durante décadas, en la mayoría de los páramos de Colombia, la producción de papa y el ganado doble propósito (carne y leche) fueron el pilar de economía familiar campesina.

Los cultivos de papa son fuente de empleo en la zona rural y, a su vez, permiten la conformación de redes de producción, procesamiento y mercadeo; además, vinculan actividades económicas como los jornales, actividad que incide de manera directa y positiva en las familias (5).

El páramo de Rabanal, ubicado en la cordillera Oriental colombiana, en los departamentos de Boyacá y Cundinamarca, comprende los municipios de Samacá, Ventaquemada, Guachetá, Lenguazaque, Ráquira y Villa Pinzón; a través del tiempo, se ha visto afectado por la disminución de áreas naturales, lo cual se traduce en pérdida de bosques y aumento acelerado de áreas para la producción de cultivos.

A pesar del gran valor ecosistémico y cultural, el páramo se ha visto influenciado de manera nega- tiva por el manejo irracional e insostenible de los pobladores, en actividades como agricultura, ganadería y minería, que han puesto en peligro crítico la diversidad florística y faunística que lo caracteriza, así como la oferta de bienes y servicios ambientales que lo hacen tan importante.

El inadecuado manejo de estos cultivos, específicamente de papa, en áreas de páramo en Boyacá se convierte en el principal problema ambiental que afecta el recurso hídrico, ocasionado por el indiscriminado uso de agroquímicos y tóxicos residuales en las zonas de nacimiento de agua (6), procesos que afectan de manera directa la salud humana.

Así mismo, el alquiler de la tierra para la producción de papa y forraje, el uso de insumos agroquímicos y los medios agresivos de labranza del suelo se oponen a la voluntad de protección del páramo, la biodiversidad y los recursos hídricos, adicionalmente; la política no es favorable a la agricultura en este ecosistema y se centra en la creación de áreas protegidas, libres de las actividades productivas (7).

La inexistente capacitación y el desconocimiento real de los pobladores hacia la conservación y uso sostenible de los recursos naturales es un problema latente; los impactos ambientales negativos sobre el páramo son cada vez más evidentes, la población de páramo desarrolla sus actividades agropecuarias sin darle el valor al territorio que ocupan.

El documento "Formulación plan de manejo del Macizo Páramo de Rabanal", elaborado por el Instituto Alexander von Humboldt, contempla entre sus objetivos la conversión de prácticas agropecuarias convencionales en sistemas productivos sostenibles menos perjudiciales al medioambiente y más rentables desde una visión financiera, además, advierte que las inadecuadas prácticas agropecuarias y el avance de la frontera agrícola sobre las áreas de páramo son responsables del desecamiento de humedales, de contaminación de aguas, destrucción de la biodiversidad, extinción de especies, erosión y daño a los suelos (8); es por esto que, según el actual Plan de Desarrollo 2014- 
2018, en las áreas delimitadas como páramos no se podrán adelantar actividades agropecuarias ni de exploración o explotación de recursos naturales no renovables, ni construcción de refinerías de hidrocarburos (9).

Ante este panorama, se plantea estructurar una propuesta participativa de reconversión de los procesos de producción agrícola actuales, que vinculen a la comunidad como actor importante en su territorio, partiendo de un diagnóstico socio-ambiental y teniendo en cuenta la agroecología como una alternativa de producción y conservación en el páramo, mediante el manejo sostenible tanto de los recursos naturales como de las áreas productivas, con la propuesta por la recuperación estructural y funcional de este ecosistema afectado, y la realización de planes de trabajo orientados hacia la mitigación del impacto ambiental y sus consecuencias, a fin de lograr el mantenimiento de la dinámica natural del ecosistema y de su entorno.

Si se considera lo descrito por (10), cualquier proceso de conversión de sistemas de producción agropecuaria convencional hacia sistemas agroecológicos debe componerse de tres fases:

1. Eliminación de insumos externos.

2. Sustitución de insumos químicos externos al sistema de producción.

3. Rediseño de los agroecosistemas con una infraestructura diversificada y funcional, fase correspondiente a la verdadera conversión agroecológica.

Y dentro del proceso de reconversión (11) se plantean realizar los siguientes procesos durante la ejecución de las tres fases, así: 1) Aumento de la biodiversidad en los subsistemas vegetal y animal, 2) Aumento de la biomasa y de la materia orgánica en el suelo, 3) Disminución de los niveles de residualidad de pesticidas, y 4) Establecimiento de relaciones funcionales entre los diferentes componentes del sistema y rediseño predial.
El proceso de reconversión de actividades agrícolas convencionales hacia la agroecología vincula a los actores del páramo de Rabanal en un esquema de sensibilización y capacitación en el cual se promuevan los principios agroecológicos, como base para la conservación de la biodiversidad en el páramo y la protección del ecosistema.

El fortalecimiento del capital social de los hogares rurales, a través de la participación en actividades de capacitación, promueve una relación de complementariedad con el capital humano y cultural, una vez que los hogares amplíen su nivel de conocimiento y mejoren sus capacidades para hacer frente a los cambios (12).

Por lo anterior, se plantea realizar el proyecto de investigación "Diagnóstico socio-ambiental de la producción agrícola en el páramo de Rabanal (Colombia)" como base para su reconversión agroecológica.

\section{Materiales y métodos}

La metodología para elaborar la propuesta participativa dentro del proyecto de investigación se desarrolla a partir del análisis del entorno en los dos sectores del área objeto de estudio: Matanegra y San José de Gacal, vereda Montoya, del municipio de Ventaquemada, Boyacá (Colombia). Se realizó una encuesta semiestructurada, con el propósito de caracterizar el grupo de pobladores campesinos, y con el fin de hacer una caracterización socio-ambiental del área, se entrevistaron 165 de los 174 predios identificados por medio de visitas finca por finca; los 9 restantes estaban deshabitados o sus ocupantes se rehusaron a entregar información.

Posterior a esta caracterización se seleccionó el grupo de pobladores asentados en el área de amortiguación del páramo, con los cuales se aplicó la metodología adaptada MESMIS (13) y en conjunción con la propuesta de estandarización y ponderación de indicadores del nivel de sustentabilidad de Sarandón (14). 


\section{Población y muestra}

La población está conformada por los habitantes de los sectores San José del Gacal y Matanegra de la vereda Montoya, municipio de Ventaquemada.
Para realizar el muestreo estratificado, inicialmente se aplicaron tres filtros:

Filtro uno: número de predios encuestados. De 174 predios identificados, se pudieron encuestar 165, en razón a que en 9 de ellos no proporcionaron información, como se muestra en la Tabla I.

Tabla I. Encuestas realizadas

\begin{tabular}{|c|c|c|c|}
\hline Lugar & Encuestas realizadas & $\begin{array}{c}\text { No proporcionó } \\
\text { información }\end{array}$ & Total predios \\
\hline San José del Gacal & 106 & 3 & 109 \\
\hline Matanegra & 59 & 6 & 65 \\
\hline Total & 165 & 9 & 174 \\
\hline
\end{tabular}

Filtro dos: Predios interesados en participar en el proyecto. De las 165 encuestas realizadas, 128 ocupantes de predios están interesados en hacer parte del proyecto (Tabla II).

Tabla II. Predios interesados en participar

\begin{tabular}{|c|c|}
\hline Lugar & Número de hogares \\
\hline San José del Gacal & 82 \\
\hline Matanegra & 46 \\
\hline Total & 128 \\
\hline
\end{tabular}

Filtro tres: Predios propios interesados en participar en el proyecto. De los 128 predios interesados en hacer parte del proyecto, 100 son propios $y$, en consecuencia, sus dueños pueden disponer de ellos para el desarrollo del proyecto (Tabla III).

Tabla III. Predios propios

\begin{tabular}{|c|c|}
\hline Lugar & Número de predios \\
\hline San José del Gacal & 67 \\
\hline Matanegra & 33 \\
\hline Total & 100 \\
\hline
\end{tabular}

Filtro cuatro: Actividad económica desarrollada en el predio. Setenta y siete de los 100 predios propios dispuestos a participar están dedicados a la agricultura (Tabla IV).
Tabla IV. Predios dedicados a la agricultura

\begin{tabular}{|c|c|}
\hline Lugar & Número de hogares \\
\hline San José del Gacal & 52 \\
\hline Matanegra & 25 \\
\hline Total & 77 \\
\hline
\end{tabular}

\section{Tamaño de la muestra}

Pasos:

1. Tamaño de la muestra para la población finita y conocida: Según metodología de la investigación (15).

Aplica la fórmula:

Donde:

$$
\mathrm{n}=\frac{\mathrm{NZ} \mathrm{Z}^{2} \mathrm{pq}}{\mathrm{i}^{2}(\mathrm{~N}-1)+\mathrm{Z}^{2} \mathrm{pq}}
$$

$\mathrm{n}$ = tamaño de la muestra

$\mathrm{N}=$ tamaño de la población

$\mathrm{Z}$ = valor correspondiente a la distribución de gauss; llamado también nivel de confianza.

$\mathrm{i}=$ error que se prevé cometer

$\mathrm{p}=$ prevalencia esperada del parámetro $\mathrm{a}$ evaluar

$\mathrm{q}$ = proporción de la población de referencia que no presenta el fenómeno en estudio (1 -p), (Tabla VI). 
Tabla VI. Valores establecidos para calcular $\mathrm{n}$

\begin{tabular}{|c|c|}
\hline$N$ & 77 \\
\hline$Z$ & 1,645 \\
\hline$d$ & 0,1 \\
\hline
\end{tabular}

Para el cálculo de p:

Se tomó una muestra piloto de 15 fincas, en las cuales se evaluó la variable 'uso de productos agroquímicos para el manejo de sus cultivos'; trece fincas respondieron que sí utilizaban productos de síntesis química para el manejo de cultivos.

$$
\begin{gathered}
p=\frac{13}{15}=0.86 \\
q=1-0.86=0.13
\end{gathered}
$$

Reemplazando en la fórmula:

$$
\begin{gathered}
\mathrm{n}=\frac{77 * 1.645^{2} * 0.86 * 0.13}{0.1^{2}(77-1)+1.645^{2} * 0.86 * 0.13} \\
\mathrm{n}=22
\end{gathered}
$$

La muestra representativa con la que se dio inicio al proyecto fue de $n=22$.

Para cada uno de los predios seleccionados se evaluó 'análisis de calidad del suelo y salud del cultivo', este análisis de hizo de manera participativa con los agricultores del páramo, quienes comprendieron conceptos agroecológicos importantes para la verificación del estado actual de sus predios, y el camino por seguir hacia una conversión agroecológica del sistema productivo; este proceso se realizó con la participación activa de los campesinos hacia la búsqueda de un cambio de actitud frente al manejo de los recursos naturales, y sensibilización frente a la concepción actual en el manejo convencional de la producción agrícola que busca aumento en la producción y rendimientos econó- micos, desconociendo las consecuencias de este manejo sobre la salud humana y el ecosistema.

Con este grupo de pobladores se desarrollaron capacitaciones en temáticas de identificación de impactos ambientales, recolección y propagación de semillas, huertas caseras, estrategias agroecológicas, agroecosistemas, producción de abonos orgánicos, elaboración de caldos trofobióticos, manejo de cercas vivas, cultivo de papa y especies representativas de la zona, especies nativas, bosques maderables y técnicas de cultivos amigables con el medioambiente.

\section{Resultados y discusión}

Los resultados del procesamiento de las 165 encuestas semiestructuradas aplicadas, respecto al análisis económico, ambiental y social de la comunidad, señalan, de manera general, que esta considera que participar en el proyecto permitirá mejorar la forma de producción, su salud y alimentación, e impactar en menor medida el ecosistema de páramo, mediante el manejo adecuado de los recursos naturales.

La caracterización socioeconómica y ambiental permite observar que los pobladores de los sectores Matanegra y San José del Gacal, de la vereda Montoya, en el municipio de Ventaquemada, centran su actividad económica en la agricultura de papa, en la cual utilizan productos de síntesis química para el control fitosanitario del cultivo; esto evidencia la prevalencia del monocultivo de papa, lo cual lleva al aumento de problemas fitosanitarios y a la pérdida de seguridad y soberanía alimentaria (Fig. 1).

Pero si bien la agricultura representa la actividad económica principal de estos campesinos, la ganadería es un renglón de importancia y ha ampliado su frontera, interviniendo los componentes de flora y fauna $y$, de manera directa, ocasionando pérdida de cobertura vegetal y biodiversidad en los predios. 


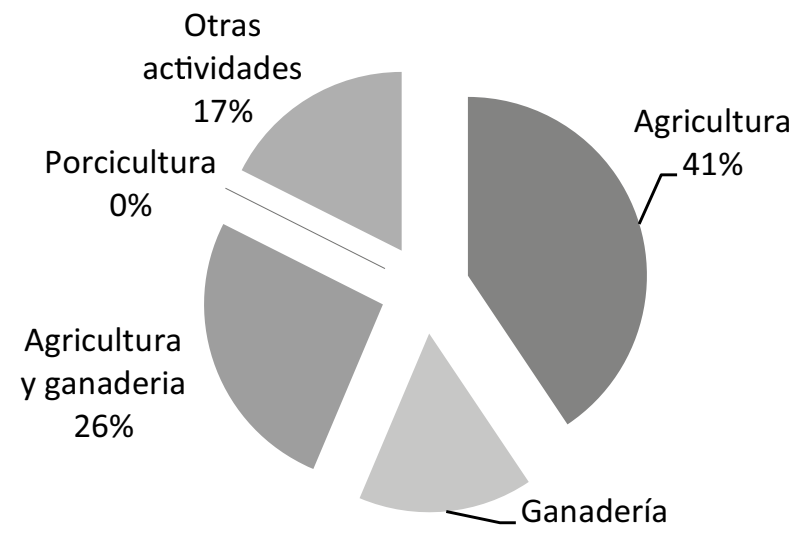

Figura 1. Principal actividad económica, sectores Matanegra y San José del Gacal.

En el proceso de acondicionamiento de los suelos de páramo para la agricultura o la ganadería se aplican enmiendas que producen un efecto en las propiedades químicas del suelo, generando cambios drásticos en la acidez y relación de bases, convirtiéndose en limitante. Una vez la vegetación de páramo es reemplazada por cultivos, el pastoreo se concentra en áreas cada vez más pequeñas y a mayor altura. El avance de la frontera agrícola y pecuaria hace que la vegetación nativa desaparezca y el suelo se erosione (16).

Aproximadamente el $92 \%$ de los encuestados relacionan la aplicación de agroquímicos en los cultivos y pastizales con las diferentes plagas y enfermedades presentes en sus predios, y mencionan, además, la aplicación de herbicidas en la preparación del suelo y en la precosecha, lo cual se observa en la Fig. 2.
No

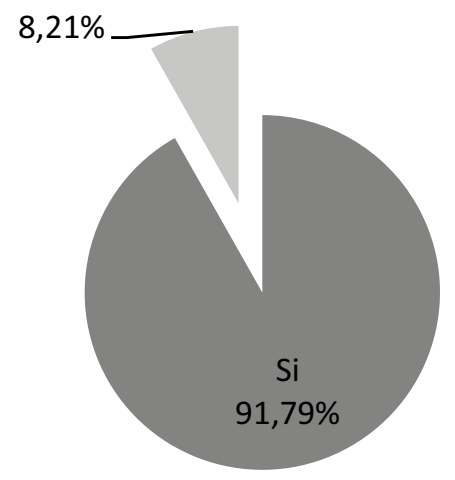

Figura 2. Uso de agroquímicos por los agricultores, sectores Matanegra y San José del Gacal.

Para el caso de Boyacá, la extensión del cultivo de la papa sobre los páramos del departamento se ha venido convirtiendo en el principal problema ambiental que afecta el recurso hídrico, debido al uso intensivo de agroquímicos y a los tóxicos residuales o biocidas en zonas de nacimiento de agua, sobre las cuales se han detectado niveles altamente peligrosos (17).

Es así como los cultivadores de los sectores objeto de estudio aplican insumos de síntesis química para la fertilización de suelo, y herbicidas, fungicidas e insecticidas altamente tóxicos para el control fitosanitario, lo cual afecta el recurso suelo y disminuye su potencial productivo natural y la salud humana. Esta alta dependencia de insumos para el control fitosanitario y para la fertilización de suelos es en ocasiones innecesaria. Los insumos externos también incluyen alimentos comprados, debido a la ausencia de huertas en la mayoría de las fincas (18). 
El manejo dado por los agricultores a los plaguicidas y a los empaques y envases de estos no es el adecuado, en términos de almacenamiento, recolección y disposición, poniendo en riesgo la salud humana, el medioambiente y los recursos naturales, al no seguir ninguna recomendación técnica, como las consagradas en la resolución 693 de 2007 (MAVDT) para el plan de gestión y devolución de los productos posconsumo de plaguicidas (19).

La pérdida de seguridad y soberanía alimentaria se encuentra relacionada con lo anteriormente descrito, en razón al sometimiento económico y tecnológico de los campesinos frente a la alta dependencia de los insumos externos.
Mediante una estrategia participativa con los agricultores se realizó la evaluación de la calidad del suelo y de la salud del cultivo, a través del análisis de parámetros indicadores en cada uno de los predios, encontrando de manera general buenas características y un estado ideal de los agroecosistemas.

Es así como se observa un estado ideal para el análisis de calidad de suelo frente a profundidad del suelo, estado de residuos, color, presencia de materia orgánica, retención de humedad y desarrollo de raíces, como lo muestra la Fig. 3. Esto indica las cualidades de los suelos en la zona objeto de estudio, en donde se encontraron suelos con capa vegetal que oscila entre $70 \mathrm{~cm}$ y $120 \mathrm{~cm}$.

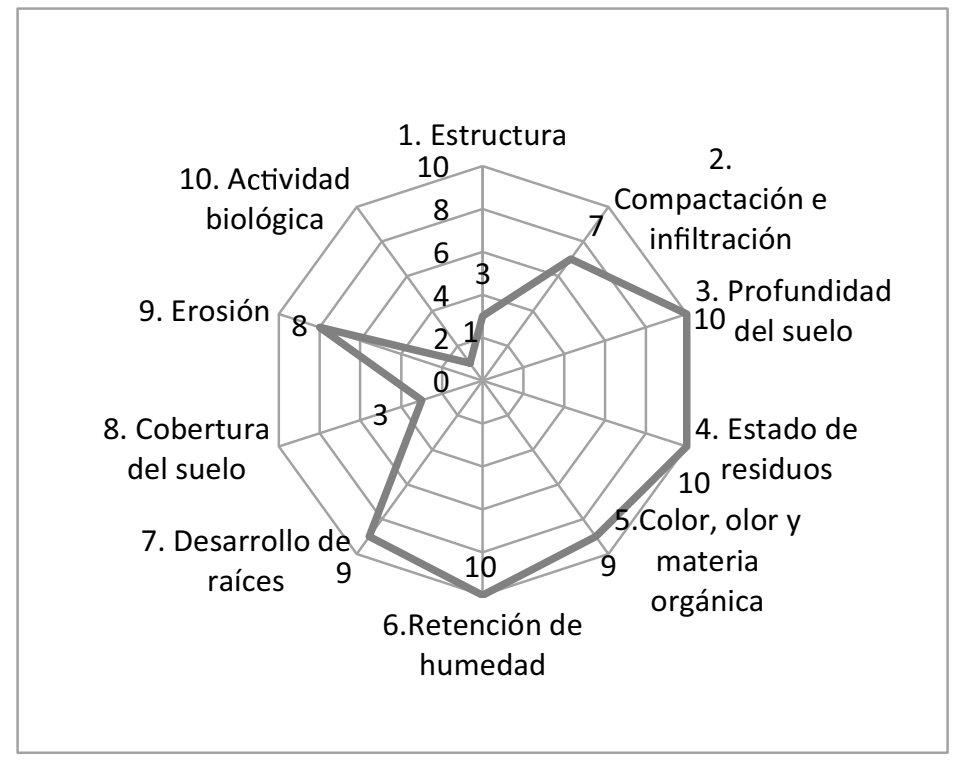

Figura 3. Calidad del suelo agroecosistemas páramo de Rabanal.

De igual manera, se muestran los resultados obtenidos frente a los parámetros cobertura, estructura y actividad biológica del suelo, encontrando valores por debajo de 5, es decir, bajo el umbral de sostenibilidad. Se evidencia una relación directa entre pérdida de cobertura y actividad biológica, lo cual obedece, probablemente, a la aplicación de agroquímicos causantes de pérdida de macroorganismos, y al suelo desnudo, que favorece la disminución de la actividad biológica; así mismo, el sobrelaboreo en los predios contribuye a la pérdida de estructura del suelo, ya que este es utilizado en todas las épocas del año para el establecimiento del cultivo de papa.

En esta evaluación se observa que con valores por debajo del umbral de sostenibilidades necesario realizar un manejo que permita mejorar los aspectos en los cuales los indicadores muestran valores bajos. Los valores de los indicadores son fáciles de observar al graficar los resultados de cada agroecosistema en una figura tipo "ameba"; esto permite visualizar el estado general de la calidad del suelo o la salud del cultivo, considerando que mientras 
más se aproxime la "ameba" al diámetro del círculo (valor 10) más sostenible es el sistema, de acuerdo con (20).

En cuanto al análisis de salud del cultivo de papa (Fig. 4), se destaca un valor importante frente a diversidad natural circundante, lo cual corrobora la presencia de especies nativas pertenecientes a bosque alto andino en la zona, lo que favorece las interrelaciones ecológicas benéficas para el agroecosistema.

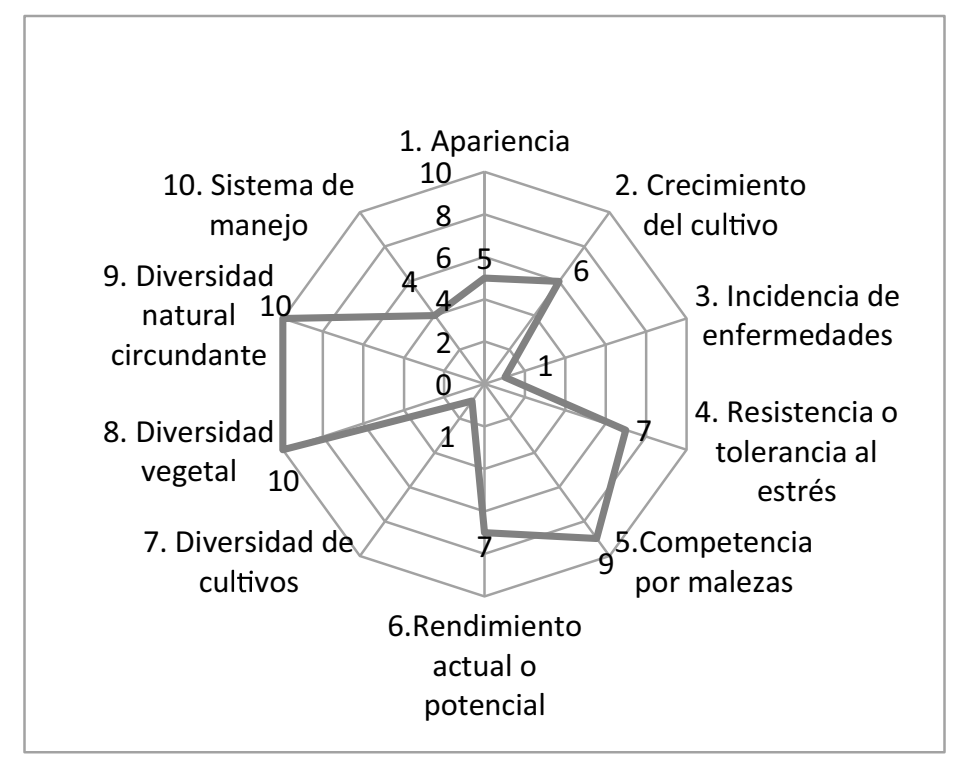

Figura 4. Salud del cultivo agroecosistemas páramo de Rabanal.

Para el parámetro incidencia de enfermedades se observa un estado fitosanitario adecuado en el cultivo, lo cual está relacionado con la aplicación de agroquímicos; sin embargo, al observar la competencia por malezas se evidencia que es alta, debido, probablemente, a la resistencia de algunas arvenses o malezas a los herbicidas aplicados para su control.

Teniendo en cuenta los indicadores apariencia, crecimiento del cultivo y sistema de manejo de este, los valores son bajos, en razón a las prácticas convencionales utilizadas en estos agroecosistemas, lo cual muestra la dependencia creciente de insumos agroquímicos, lo que afecta de manera negativa el ecosistema.

Es importante destacar la poca diversidad de cultivos con que cuentan los predios, lo cual impide a la población tener acceso a alimentos que podrían cultivar en el agroecosistema, perdiendo así seguridad y soberanía alimentaria.

\section{Conclusiones}

Al analizar los agroecosistemas frente a calidad del suelo y salud del cultivo, se evidenció que, no obstante, los suelos de páramo tienen un porcentaje de materia orgánica importante, y que la biota del suelo ha sido afectada por la aplicación de productos de síntesis química, responsables de pérdida de biodiversidad. De igual manera, se observan cultivos sanos por el manejo químico para el control fitosanitario del cultivo.

En cada uno de los predios evaluados se observa la necesidad de implementar prácticas menos contaminantes en favor de la conservación del recurso hídrico, del suelo y de la vegetación nativa, prácticas relacionadas con el manejo agroecológico en cada uno de los predios.

Actualmente, la estimación de la sostenibilidad de los agroecosistemas es una preocupación prioritaria de muchos investigadores agrícolas que han 
propuesto diferentes atributos para evaluar la productividad, estabilidad, resiliencia y adaptabilidad de agroecosistemas (21).

Para el área objeto de estudio se plantea la vinculación de los campesinos en un proceso de capacitación en el cual se propicie el intercambio de conocimientos y experiencias, el rescate de saberes y prácticas, el reconocimiento de las necesidades del sistema de producción, la identificación de alternativas y la adaptación de tecnologías menos contaminantes, en busca del aprovechamiento y manejo sostenible de los recursos naturales hacia la diversificación de la producción y la sostenibilidad de los agroecosistemas (22).

Para el manejo de la fertilidad del suelo se utilizan varios principios agroecológicos que incluyen la elaboración de abonos compostados (humus, compost, lombricompuesto y bokashi), principios que promueven importantes procesos de regulación de nutrientes y de materia orgánica, que inciden tanto en la estabilidad general del agroecosistema, como en sus posibilidades de manejo de organismos patógenos (23).

Se plantea la necesidad de implementar prácticas agroecológicas en cada uno de los predios que permitan el establecimiento de huertas dentro de un concepto de diversificación de cultivos y asociado a un manejo agroecológico a fin de prevenir impactos sobre el ecosistema y la salud humana.

Esta propuesta participativa de conservación de los recursos naturales desde la agroecología debe buscar la sostenibilidad ambiental y el beneficio social, aspectos importantes en la actividad agrícola en el páramo de Rabanal.

\section{Agradecimientos}

-Ecopetrol

-Grupo de investigación Sistemática Biológica (SisBio), Universidad Pedagógica y Tecnológica de Colombia.

-Sociedad Científica Latinoamericana de Agroecología (SOCLA)

\section{Referencias}

(1) Etter A. y van Wyngaarden W. Patterns of landscape transformation in Colombia, with emphasis in the Andean Region, Ambio, 29: 432-439. CP: Arvey A.C. y Sáenz G.J. Evaluación y conservación de biodiversidad de paisajes fragmentados de Mesoamérica. 2007.

(2) Etter A. Mapa general de ecosistemas de Colombia. Informe nacional sobre el estado de la biodiversidad. Instituto de Investigación de Recursos Biológicos Alexander von Humboldt. Bogotá, vol. 1, 1998.

(3) Lozano F, Mendoza J, Vargas A, Caycedo P, Vargas $W$, Jiménez $E$, Aristizábal S, Ramírez D, Murillo X, y Ríos C. Modelo de manejo sostenible de paisajes rurales para la conservación de la biodiversidad en la región andina colombiana, Instituto de Investigación de Recursos Biológicos Alexander von Humboldt, Bogotá, Colombia, 2006.

(4) Van der Hammen T. Diagnóstico, cambio global y conservación. Congreso Mundial de Páramos 2002; 1: 60-71.

(5) Ministerio de Ambiente, Vivienda y Desarrollo. Dirección de Desarrollo Social y Sostenible. Federación de Producción de Papa. Guía ambiental para el cultivo de papa. 56 pp. 2004.

(6) Avellaneda A, y Jaramillo C. Alteración del Páramo de Chontales en Boyacá por ganadería y aplicación de plaguicidas en papa. Memorias Congreso Mundial de páramos, CAR, IDEAM y Conservación Internacional 2002; 2: 812-818.

(7) Robineau O, Chatelet M, Soulard C, Michel D, y Posner J. Análisis de las prácticas productivas en el área circundante del páramo de Rabanal: aportes para la conservación de los recursos naturales y modalidades de aplicación de estas prácticas. Avances en investigación para la conservación de los páramos andinos, Condesan. 19 p. 2014.

(8) Instituto de Investigación de Recursos Biológicos Alexander von Humboldt, Corporación 
Autónoma Regional de Cundinamarca, Corporación Autónoma Regional de Boyacá, Corporación Autónoma de Chivor. Estudio sobre el estado actual del macizo del páramo de Rabanal. Bogotá D. C., 2008.

(9) Presidencia de la Republica de Colombia. Plan Nacional de Desarrollo 2014-2018. Disponible en: http://wp.presidencia.gov.co/ sitios/normativa/leyes/Documents/LEY\% 20 $1753 \% 20$ DEL\%2009 \%20 DE \% 20JUNIO \%20DE\%202015.pdf

(10) Altieri M. y Nicholls C. Cultivos de cobertura y utilización de Mulch. Agroecología: bases científicas para una agricultura sustentable. Editorial Nordan Comunidad Montevideo, 1999; 10: 203-215.

(11) Altieri M. Bases agroecológicas para una producción agrícola sustentable. Agricultura Técnica, 1994; 54: 371-386.

(12) Bermúdez M. Determinación de indicadores agroecológicos en sistemas agroforestales y de medios de vida de fincas cafeteras de Colombia, Costa Rica y Nicaragua. Tesis de maestría. CATIE, Turrialba, Costa Rica, 2007.

(13) Masera O., Astier M. y Galván Y. Formulación de indicadores socioambientales para evaluaciones de sustentabilidad de sistemas de manejo complejos. En:Evaluación de sustentabilidad. Un enfoque dinámico y multidimensional, Ed.Imaglmpressions 2008; 73-94.

(14) Sarandón S. El desarrollo y uso de indicadores para evaluar la sustentabilidad de los agroecosistemas. En:Sarandón S.J. (ed),Agroecología. El camino hacia una agricultura sustentable. Ediciones Científicas Americanas, 2002; 20: 393-414.

(15) Rodríguez M. Metodología de la investigación. México: Universidad Juárez Autónoma de Tabasco, 2005.

(16) Hofstede R, Segarra P. y Vasconez P. Los páramos del mundo. Global PeatlandInitiative/NCIUCN/ Ecociencia. Quito. 2003, p. 299. CP: Estupiñán L, Gómez J, Barrantes, V, y Limas L.
Efecto de actividades agropecuarias en las características del suelo en el páramo el granizo (Cundinamarca - Colombia). Revista U.D.C.A Actualidad \& Divulgación Científica, 2009; 12: 79-89.

(17) Avellaneda A. Gestión y políticas para una gestión ambiental de plaguicidas en Colombia 1988; 254-256. En: Primer Simposio Internacional y Segundo Nacional Plaguicidas, Ambiente y Salud Humanas. Rapalm, UN-Sena. Palmira, 1998.

(18) Calderón P. y Flórez G. Valoración y análisis de indicadores de sostenibilidad en seis unidades de producción agropecuaria de la cuenca media del río Chinchiná. Revista Luna Azul, 2014; 41: 7388.

(19) Monroy O. Caracterización de las prácticas agrícolas asociadas con el uso y manejo de plaguicidas en cultivos de papa. Caso vereda Mata de mora, en el páramo de Merchán, Saboyá, Boyacá. Tesis de maestría. Pontificia Universidad Javeriana, Bogotá, Colombia, 2009.

(20) Altieri M. y Nicholls C. Un método agroecológico rápido para la evaluación de la sostenibilidad de cafetales. Manejo Integrado de Plagas y Agroecología (Costa Rica), 2002; 64:17-24.

(21) Masera O., Astier M. y López S. Sustentabilidad y manejo de recursos naturales: el marco de evaluación MESMIS. México:Mundiprensa. 1999.

(22) Calderón P., Tobasura I. y Miranda J. Planificación participativa de fincas de aprendizaje: el caso de Colombia. En: C. Villanueva, C. J. Sepúlveda y Muhammad Ibrahim (Eds.), Manejo agroecológico como ruta para lograr la sostenibilidad de fincas con café y ganadería (29-50). Turrialba, CR: CATIE, 2011.

(23) Varela M. Evaluación de sistemas de producción agroecológicos incorporando indicadores de sostenibilidad en la sabana de Bogotá. Tesis de maestría. Pontificia Universidad Javeriana. Bogotá, Colombia, 2010. 